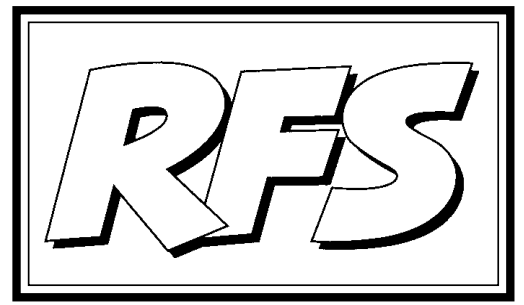

Revista de Fomento Social, 54 (1999), 507-518

\title{
Relaciones entre las ONGD del Norte y las ONGD del Sur
}

Desde su privilegiada atalaya boliviana, el autor analiza los problemas más frecuentes que se presentan en las relaciones entre ONGD del Norte y del Sur. Aboga, entre otras cosas, por un compromiso estable entre ambas.

\section{Introducción}

Las ONGD del Norte y las ONGD del Sur se necesitan mutuamente. Las unas sin las otras no podrían cumplir su misión de servicio a los pueblos del SUR y de construcción de un mundo solidario. Pero sus relaciones tienen puntos críticos, que intento explicitar en la primera parte, partiendo de mi experiencia de 24 años en varias ONGD de Bolivia. En la segunda parte expongo mi punto de vista sobre

(*) Jesuita. Ha trabajado durante varias décadas en Bolivia con especial dedicación a tareas de desarrollo con comunidades rurales e indígenas. 
las prioridades que deben tener las ONGD hoy en Bolivia. Creo que las prioridades que van surgiendo en este país pueden visualizar el cambio operado en toda Latinoamérica desde la época de las dictaduras militares.

\section{Puntos críticos}

\section{1. ¿Quién decide lo que se va a hacer?}

Las ONGD del Sur sienten que no pueden hacer lo que creen que sería más necesario para sus poblaciones, sino únicamente lo que las del Norte están dispuestas a financiar. Hace unos años había muchas menos ONGD tanto en el Norte como en el Sur y se podía tener una relación más libre y dialogante entre unas y otras. Ahora hay una enorme saturación de ONGD y de proyectos. Los «oficiales» de las ONGD del Norte tienen que recibir, procesar, presentar, acompañar y evaluar tal cantidad de proyectos que es imposible que puedan analizar bien todos ellos. Por eso las prioridades que señalan las ONGD del Norte se aplican con menos flexibilidad que hace unos años. Y no es que entonces la situación fuese la ideal.

Desde el Sur reivindicamos cambios estructurales que vayan pasando por lo menos algo del poder de decisión al Sur. No pretendemos que las ONGD del Sur seamos juez y parte. Pero sí que la opinión del Sur tenga cauces institucionales para participar en la toma de decisiones sobre la Cooperación Internacional.

Se han dado varios avances en esta dirección. Algunas ONG del Norte han contratado algún personal fijo en cada país del Sur en que tiene proyectos. Otras han establecido plataformas de análisis de algún punto concreto (p.e.: desarrollo sostenible, coyuntura política) en las que participan sus contrapartes del Sur. Con estas medidas las ONG del Norte tienen mejor información, pero en realidad no se puede hablar de una real participación del Sur en la toma de las decisiones sobre la cooperación que reciben.

Creo que el próximo paso debe ser la creación de Consejos en los países del Sur, que ayuden a fijar prioridades y tal vez a analizar los objetos de financiamiento (proyectos, programas, grupos, instituciones). Pero evidentemente no sería conveniente ni operativo que cada ONGD del Norte crease su Consejo en cada uno de los países del Sur donde tiene contrapartes. Este paso sólo se puede dar en la medida en que maduren los procesos de formación de redes de ONGD, que 
ya se están desarrollando en el Norte.

Si las ONGD del Sur se ven condicionadas por las prioridades que establecen las ONGD del Norte, éstas tampoco pueden financiar siempre lo que a ellas les parece más conveniente. Están condicionadas por sus fuentes de financiamiento: el público que responde a sus campañas (más sensible a las emergencias y a los proyectos de gran visibilidad material) y los organismos públicos de cooperación, que también tienen sus exigencias.

\section{2. ¿Debemos hacer todos lo mismo?}

Las del Norte tienen prioridades muy similares entre sí, lo que hace difícil lacada vezmás necesaria especialización de las del Sur. Al final todos hacen casi lo mismo. Proliferan ONGD dedicadas a niños de la calle, a pequeños grupos productivos femeninos, a la salud reproductiva, a los derechos de las mujeres y a todo lo que tenga relación con la ecología. Entre las ONGD de Bolivia es común decir, medio en broma, medio en serio: -Si quieres asegurar la plata, haz un proyecto ecológico de mujeres indígenas.

Estas actividades son necesarias, pero junto a ellas hay otras prioridades de mucha trascendencia para las que no se encuentra el suficiente apoyo. Las que a mí me parecen más importantes hoy en Bolivia (que expongo en la segunda parte de esta comunicación) resultan muy difíciles de financiar, porque no entran en las prioridades del Norte.

Cierto que hace ya algún tiempo que se ven avances, especialmente en planteamientos conjuntos para el desarrollo de una zona (cuenca, microrregión, barrio) de varias ONG del Sur -cada una con su especialidad-que se unen a organizaciones populares y oficiales, y reciben apoyo de varias ONG del Norte. Con esto se consigue más coherencia, mejor coordinación y mayor cobertura y diversidad de los proyectos. Pero percibo poca flexibilidad y receptividad en el Norte ante proyectos muy importantes que se salgan de las prioridades prefijadas.

\subsection{Control y burocracia}

Las ONGD del Sur se ven sometidas a exigencias contradictorias: por una parte cada vez se les exigen mayores controles administrativos, métodos más sofisticados de planificación, seguimiento y evaluación y una presentación de sus proyectos 
cada vez más complicada. Si un proyecto que presentamos a una ONGD del Norte va a ser cofinanciado por la Unión Europea o por otras instituciones públicas, su elaboración es tan laboriosa como la de una tesis doctoral.

Para cumplir estas exigencias necesitan cada vez más personal y mayor competencia profesional. Pero cada vez hay mayor reticencia en las del Norte para financiar sueldos y gastos administrativos.

Las del Norte ven con justificada alarma la proliferación de ONGD en el Sur y el crecimiento desmesurado de algunas de ellas. Perciben que muchas ONGD del Sur han nacido, se mantienen y crecen más para atender las necesidades de su personal que para atender las necesidades de la población a la que se quiere ayudar. Y es cierto que en países como Bolivia hay una gran escasez de puestos de trabajo para profesionales y las ONGD representan muchas veces una excelente solución a la necesidad personal de tener y mantener un trabajobien remunerado. Es significativo que frente a las 285 ONGD que hay en España, en Bolivia ya en 1991 habíamos catalogado más de 600.

\section{4. «Estrategia de intervención»: plazos para obtener resultados}

Las ONGD del Norte exigen resultados a corto plazo. Recuerdo el estupor de un oficial de proyectos de una ONGD centroeuropea cuando le dije que nuestra perspectiva de trabajocon los guaraní-chiriguanoera de por lomenos cincuenta años (llevamos ya 34), y que plantear un proceso más corto me parecía una irresponsabilidad. El me estaba planteando una «estrategia de intervención» de tres años.

Pienso que a veces se tiene una visión muy simplista de los problemas del desarrollo. En el caso que estábamos tratando nos referíamos a una etnia muy golpeada por la colonia y por la sociedad criolla. Los guaraní-chiriguano están en un franco proceso de recuperación de su identidad, de creación de instituciones propias y de sustitución del alienante trabajo en la zafra azucarera por una producción agropecuaria propia. Existe una fundada esperanza de que puedan vivir la inevitable globalización e inserción en la economía de mercado sin perder todos sus valores culturales.

Pero para llegar a la situación actual se han necesitado decenios de convivencia y diálogo, cantidad de tentativas, unas exitosas, otras no tanto y varios fracasos estrepitosos. Se ha precisado también mucho estudio histórico, antropológico, sociológicoy lingüístico, mucha experimentación agropecuaria, muchos proyectos 
agropecuarios pequeños, medianos y grandes; todo un plan de educación intercultural y bilingüe; más de trece pleitos de tierras con hacendados, etc., etc. Y todavía queda mucho por hacer.

Mirespuesta, algo desafiante, a mi interlocutoral plantearlemediosiglodeactuación quería decirle:-¿queremos hacer algo serio por los guaraní-chiriguano o queremos poner paños calientes?; y es que la eficacia cortoplacista suele estaren contradicción con la eficacia a largo plazo y con los necesarísimos cambios estructurales.

Es verdad que la ineficiencia de las ONGD del Sur se puede arropar en la necesidad de los largos plazos, pero los problemas de desarrollo son tan complejos y de tan difícil solución, que sólo pueden solucionarse con amplias perspectivas de tiempo, de colaboración interinstitucional y de competencia profesional en múltiples disciplinas.

\subsection{Exigencia de éxito}

La probabilidad de que un proyecto de desarrollo tenga éxito está en relación directa al desarrollo humano de la población a la que va dirigido. La probabilidad de fracaso es inversamente proporcional a este desarrollo humano. Pero existe una creciente presión para que los proyectos de las ONGD tengan éxitos visibles. El resultado es que las ONGD del Sur rehuyen trabajar en el desarrollo de las áreas más deprimidas, donde el éxito en términos de desarrollo permanente es muy difícil. En el mejor de los casos se dedicarán a un trabajo asistencial con alguno de estos sectores, o a la promoción individual de un escaso número de personas, porque esto sí que es financiable. Pero son muy pocas las instituciones que perseveran en la búsqueda de soluciones estructurales a los problemas del 10\% más pobre de la sociedad.

Por amarga experiencia puedo asegurar que es inútil el intento de desarrollar cooperativas agropecuarias en grupos humanos que no tienen resuelto el problema de la alimentación básica. Mucho más grave es cuando se los intenta convertir en sujeto de crédito. Se debe asegurar primero que cada familia cultive una chacra que le permita tener una canasta alimenticia elemental. Sólo entonces se puede intentar dar algún paso hacia alguna otra forma de unidad productiva asociada. Después de muchos intentos fallidos tal vez se logre encontrar un modelo que se multiplique y perdure. Es posible, pero lento y costoso. Es que poner en marcha procesos de desarrollo estables en poblaciones muy pobres es verdaderamente 
difícil y requiere de mucha paciencia y perseverancia.

Muchas veces los parámetros para valorareléxito son totalmente distintos entre la cultura de las ONGD y la cultura popular. En la cultura popular pesa mucho más lo que tiene un fuerte valor simbólico, que un resultado económico, técnico o social impecable. Uno de los fracasos más estrepitosos lo tuvimos un compañero y yo en el intento de instalar una turbina eléctrica en un pueblito llamado Toracarí. Fue algo comparable al fracaso de Zorba el Griego con su andarivel para transportar troncos del bosque al puerto. Tuvimos a todo el pueblotrabajandoduramente durante meses. Cuando por fin se inauguró se prendieron diez focos de $100 \mathrm{w}$ cada uno, que arrebataron de entusiasmo a todos los toracareños. Poco después de la inauguración, la presión del agua hizo reventar todos los tubos y provocó un desastre total, que ya no tuvo compostura. Han pasado 25 años. Toracarí sólo tiene electricidad en los días de fiesta mediante un generador a diesel.Peroel pueblode Toracaríestá orgullosísimo de la aventura de la turbina. La rueda hidráulica que la movía está como monumento en la única plaza. Para nosotros fue un gran fracaso, para ellos fue algo grande, que los enorgullece y distingue de los otros pueblos.

Pienso que el desarrollo consiste en que un grupo humano pase de ser vagón, a remolque de iniciativas que le vienen de fuera, a ser locomotora, que arrastra a cualquiera que quiera engancharse a colaborar a su desarrollo. Cuando esto ocurre -y lo he podido presenciar varias veces-es algo electrizante. Pero para que ocurra es necesario que se hayan producido muchos pequeños hechos llenos de contenido simbólico, muchos pequeños proyectos en pequeños grupos, que vayan generando confianza en sí mismos y en la ONGD que se acerca a ellos. Y esto a lo largo de años. Es necesario que perciban que la ONGD noles va a abandonar en el fracaso, que está con ellos a las duras y a las maduras. Que lleguen a tener la confianza de criticar a la ONGD de frente. Así se van creando las condiciones de posibilidad para que un buen día algún proyecto actúe como catalizador. Entonces los habitantes de toda una microrregión o cuenca o de todo un barrio se convierten en locomotora de su propio desarrollo.

Pero para poder elegir las poblaciones más deprimidas socio-económicamente y perseverar junto a ellas, a pesar de las altas probabilidades de fracaso, es necesario que también las ONGD del Sur tengan la seguridad de que sus contrapartes del Norte no les van a abandonar, que se identifiquen con ellas en su opción por los más marginados y en los riesgos de fracaso que asumen. 


\subsection{Modalidades de financiamiento}

Desde hace ya por lo menos un decenio, se habla de tres modalidades comunes de financiamiento: por proyectos, por programas y por apoyo institucional. Estas dos últimas ofrecen mayor estabilidad, pero las aplican muy pocos países y muy pocas ONGD del Norte. En realidad sólo conozco una, la holandesa NOVIB, que financie a algunas ONGD del Sur mediante apoyoinstitucional. Lamentablemente el proceso de mejoramiento de las modalidades de financiamiento parece estar no sólo detenido, sino en retroceso. El financiamiento por proyectos es la norma y las otras modalidades la excepción. Esto tiene a las ONGD del Sur en continua zozobra, pues nunca se tiene garantía de poder continuar trabajando en una zona una vez que se finalice el proyecto en curso.

Por proyectos: es la forma más común de financiamiento. Suelen ser proyectos bastante puntuales, aunque con frecuencia está previsto que se desarrollen a lo largo de tres años. Los financia una ONGD del Norte, que exige que la del Sur consiga un aporte local que financie un porcentaje del proyecto. La ONGD del Norte evalúa el proyecto, que se lleva con una contabilidad rigurosamente separada, supervisada por una auditoría financiera. Cuando la ONGD del Sur tiene muchos proyectos la administración se complica notablemente, porque cada ONGD del Norte tiene sus propias exigencias al respecto, y las evaluaciones proliferan. Con este sistema se hace muy difícil cubrir algunos gastos necesarios (p.e. de dirección y administración).

Ayuda programática: La ONGD del Sur elabora todo el programa que piensa desarrollar en un período de tiempo (normalmente un trienio), con todo su presupuesto. Un grupo de ONGD del Norte financia el programa, comprometiéndose cada una de ellas a financiar un porcentaje. Se lleva una única contabilidad, supervisada por una única auditora financiera y suele haber una única comisión evaluadora. Supone conocimiento y confianza entre todos los que intervienen del Norte y del Sur.

Apoyo institucional: Cuando ya hay una confianza muy grande y una relación muy fluida se puede acceder al apoyo institucional. La ONGD del Norte se compromete a pasar cada año una cantidad substancial a la ONGD del Sur. Recibe información permanente de su programación y de sus evaluaciones, así como de sus balances y estados de resultados económicos. 


\subsection{ONGD y Sociedad Civil}

El desarrollo de la Sociedad Civil es una cuestión clave para el desarrollo de los países del Sur. Tenemos unos Estados muy deficitarios en servicios básicos. La empresa privada es también débil. Las Universidades realizan una investigación social y técnica muy reducida, con gran escasez de medios. Unas ONGD bien arraigadas y con la suficiente autonomía financiera constituyen una parte esencial de la Sociedad Civil, llamada a jugar un papel insustituible en el desarrollo de nuestros países. Pero las cuestiones de desarrollo institucional de las ONGD, de su estabilidad y de su autofinanciamiento no encuentran suficiente apoyo en el Norte. Se exige que las ONG del Sur tengan porcentajes crecientes de financiamiento propio, pero es muy difícil que una ONG del Norte apoye un proyecto para asegurar fuentes de ingreso propios y permanentes a una ONG del Sur.

Algunas ONGD del Sur han tenido logros muy significativos. Fe y Alegría ha conseguido que las instituciones públicas financien los sueldos de la mayor parte de su personal y son famosas sus campañas de rifa. Pero por regla general las ONGD del Sur no se han caracterizado por agotar todos los medios para conseguir financiamientolocal.

El financiamiento local puede venir de varias fuentes: inversiones propias, subvenciones estatales, cobros de servicios, campañas, apoyo de la empresa privada o de la sociedad civil. Pero es muy difícil conseguir un flujo de financiamiento regular que logre cubrir una parte sustancial del presupuesto de la ONGD del Sur. Las dificultades provienen de la debilidad de las instituciones públicas y privadas de nuestros países del Sur, de la ideología de sus autoridades y funcionarios, y de la misma pobreza de la población. Las ONGD del Sur se justifican diciendo que ellas están al servicio de las poblaciones, y que ni saben, ni pueden dedicarse a buscar el financiamiento local, que les absorbería mucha energía. De todas maneras creo que las del Sur deben hacer un mayor esfuerzo y las del Norte, que tienen mucha más experiencia en «fund-raising», deben ayudarles decididamente en este empeño. 


\section{Prioridades para las ONGD en Bolivia}

\section{$1^{a}$. Educación que prepare para la realidad que se nos viene}

El 50\% de la población boliviana tiene menos de 19 años. Estamos en plena transición demográfica (boom). En los últimos 30 años la mortalidad infantil se ha reducido en un 60\% (de 243 a 96 muertes en los 5 primeros años de vida de cada mil nacidos, según el PNUD), mientras que la tasa de fecundidad sólo se ha reducido en un $33 \%$ (de 6,5 hijos por mujer a 4,36 según WPP). Actualmente el número de asalariados legales en Bolivia está alrededor de 700.000, sobre una población de siete millones que vive en su inmensa mayoría de actividades económicas familiares o individuales (agricultura, ganadería, artesanía, comercio, contrabando e infinidad de otras actividades). Las perspectivas de crecimiento de puestos de trabajo asalariados son muy reducidas. En los próximos veinte años se incorporarán a la actividad laboral unos tres millones y medio de jóvenes, que tendrán que generar su propia fuente de trabajo.

Por esto, sin dudar, pongo como primera prioridad para las ONGD desarrollar cambios pedagógicos y curriculares que equipen mejor a los/as adolescentes y jóvenes para:

- afirmar su identidad personal y cultural, con sus propios valores

- generar y mantener su propia fuente de trabajo: todos deben tener nociones de gestión, administración y mercadeo y, a ser posible, una destreza específica.

Algunos dirán que ésta es una tarea que corresponde al Ministerio de Educación y no a las ONGD. Mi convencimiento es que la Reforma Educativa en marcha, que copia muchas cosas de la Reforma Española, tiene tales dificultades de planteamiento, financiamiento y ejecución que ha perdido ya la capacidad de realizar por sí sola el gran cambio que se necesita. La situación en la que se encuentran los/las adolescentes y jóvenes bolivianos/as al terminar su educación primaria y secundaria es totalmente diferente a la de sus coetáneos españoles. Por eso los planes educativos deben partir de planteamientos diferentes.

Se necesitan experiencias educativas que sirvan de modelo para la Reforma Educativa. Fe y Alegría puede ser el gran instrumento nacional para el cambio educativo, si se le ayuda no sólo a mantener lo que tiene, sino a generar este cambio. 
También otros proyectos educativos de menor envergadura, pero orientados en esta línea, deben ser prioritarios.

\section{$2^{o}$. Apoyo a la participación popular}

La Ley de Participación Popular promulgada hace unos cinco años, fomenta el desarrollo de los pequeños municipios y de las zonas periféricas, al hacerles participardel presupuesto nacional, y establece mecanismos de control social sobre la gestión pública. Muchas funciones que antes realizaban las ONGD pueden ser ahora asumidas por los poderes locales. Esta ley es un gran instrumento para desarrollar una democracia participativa. Como todo instrumento se puede utilizar bien o mal. La incompetencia, la desidia y la corrupción han destruido en muchas alcaldías los potenciales beneficios de la Ley. Pero ha habido otras, bien asesoradas, que han logrado avances espectaculares en poco tiempo.

Los poderes locales y las organizaciones populares que deben controlarlos necesitan apoyo en diagnóstico, planificación, gestión y evaluación, y en la formación de sus líderes y funcionarios. Y las ONGD son hoy las instituciones más adecuadas y preparadas para dar este apoyo, como se demuestra en los municipios que han logrado los mejores resultados.

Quiero traer el ejemplo del municipio de Charagua, al que estoy fuertemente vinculado desde hace 25 años. Es un municipio muy extenso (unos $50.000 \mathrm{~km} 2$ ) pero poco poblado (no llega a 20.000 habitantes). Tiene un núcleo urbano de unos 3.000 habitantes, habitado en su mayoría por «karais» (no indígenas). El resto de la población es mayormente guaraní-chiriguano y vive en unas treinta comunidades (aldeas). Podemos sintetizar los logros de esta alcaldía en cuatro puntos:

- en primer lugar se ha logrado que tres de los cinco integrantes del Concejo Municipal sean guaraní-chiriguanos;

- en segundo lugar, se ha realizado un diagnóstico, que permite identificar los puntos claves para el desarrollo de la zona y un plan a largo plazo;

- en tercer lugar se han utilizado honesta e inteligentemente los recursos provenientes de la Participación Popular (unos US\$200.000) para apalancar financiamientos que multiplican esta cifra; y finalmente,

- en cuarto lugar, se aplican estrictamente los Planes Operativos Anuales, que extienden y desarrollan las vías camineras, la electrificación, las comunicaciones, los servicios de salud y educación, y apoyan la economía campesina 
con pequeños créditos para la compra de vacas.

Evidentemente para estos logros ha sido decisivo el apoyo de una ONGD con cinco lustros de trabajo en la zona.

\section{$3^{\circ}$. Presión política (lobby)}

Desde la época de las dictaduras militares hasta ahora, las relaciones de las ONGD del Sur con los organismos estatales han cambiado radicalmente y deben cambiar aún más. En tiempo de las dictaduras militares el objetivo de las ONGD era el restablecimiento de la democracia. No era posible la colaboración con las instituciones de la dictadura. La actitud era de enfrentamiento. Por la fuerza de la inercia nos ha costado mucho cambiar esta actitud en nuestras relaciones con los organismos estatales, pero, a los 18 años del restablecimiento de la democracia, ya hemos logrado desarrollar un nuevo discurso. Este discurso se podría sintetizar en la siguiente afirmación: Ya que no podemos cambiar el sistema económico y político, aprovechemos todos los resquicios que nos permitan humanizarlo.

En un país pequeño como Bolivia es posible y no demasiado complicado que propuestas legislativas elaboradas, inspiradas o asesoradas por las ONGDlleguen a presentarse en las Cámaras del Congreso y sean aprobadas. Ha sido muy significativa la participación de las ONGD en la reforma de la Constitución, que define a Bolivia como país multiétnico y pluricultural, en las leyes de Tierras y Territorios Indígenas, de Participación Popular y contra la violencia doméstica, y en el Código del Niño y de la Niña. También es muy posible influir en los ministerios, prefecturas y municipios no sólo en puntos específicos, sinoen también en las mismas estructuras administrativas: creación de la Secretarías Nacionales para problemática tales como la de los pueblos indígenas, los géneros, la participación popular, oel establecimientoen cada municipio de Defensorías del Niño y de la Niña.

De nuevo aquí se percibe una debilidad en los organismos estatales y en los partidos políticos, que deben elaborar las propuestas de ley. Normalmente las comisiones de las dos Cámaras del Congreso aceptan de buena gana la colaboración que les pueden brindar las ONGD. Pero las ONGD necesitan profesionales de alto nivel para encarar las funciones de elaborar, inspirar y asesorar las leyes y las disposiciones administrativas. 


\section{$4^{o}$. Apoyo a los MCS independientes}

El poder político y de manipulación que tienen los medios de comunicación masiva es de sobras conocido. Hay una fuerte tendencia al monopolio de estos medios por parte de grandes consorcios de la empresa privada, vinculada a las multinacionales. Es importante que haya medios independientes, que sean capaces de mantener una audiencia significativa en su zona de actuación y ser expresión de la cultura, de las voces y de los intereses populares.

En Bolivia tenemos la gran cadena Radiofónica FIDES, que logra autofinanciarse y que, por más que sea polémica y denostada por algunos sectores, mantiene su independencia. Pero se necesitan otros MCS de carácter más particular, que se expresen en alguna de las 37 lenguas que se hablan en Bolivia, o que se dirijan a una zona o sector más particular de la población, o que tengan un carácter educativo y no comercial. Los que existen actualmente llevan una existencia muy precaria y es muy difícil que se creen otros nuevos, si las ONGD del Norte no están decididas a apoyarlos a largo plazo.

\section{Conclusión}

A lo largo de estas líneas he planteado mi visión sobre las exigencias de la misión común de las ONGD del Norte y del Sur, para contribuir de verdad al desarrollo de los pueblos a cuyo servicio están. Es evidente que se debe seguir apoyando a las ONGD al servicio inmediato de pequeños sectores de población, que tienen menores requerimientos institucionales y que son verdaderos signos de vida y de esperanza. Si no abogo más por ellas, es porque creo que tienen ya el merecido aprecio del publico y de las ONGD del Norte. Pero el mayor servicio a nuestras poblaciones y nuestra misión de justicia estructural nos exigen también otro tipo de ONGD -frecuentemente satanizadas-, más difíciles de crear, mantener, controlar y financiar. Las propuestas y prioridades que he expuesto exigen que en el Sur haya unas ONGD estables, con visión del desarrollo nacional, con personal de buen nivel profesional y con buen desarrollo institucional. Exigen también que las contrapartes del Norte no practiquen con las del Sur «el amor libre», sino que adquieran compromisos estables con altos niveles de comunicación y, por supuesto, también de exigencia.

\section{RFS}

